\title{
On the methodologies and transferability of bicycle research: A perspective from outside academia ${ }^{1}$
}

\author{
Leonie Walta \\ leonie@leoniewalta.nl
}

\section{$1 \quad$ Introduction}

Driven by an increasing concern about urban liveability, climate change, and healthy life styles, amongst others, researchers are aiming to better understand why people bicycle and what could induce them to cycle more. Given the importance of local conditions and culture, there is not just one general answer to those questions. Furthermore, there is an ongoing debate on which methodological approach delivers the most meaningful results. This commentary outlines the current position of bicycle research on the question of why people bicycle and proposes ways of moving forward.

Worldwide, bicycling is increasingly regarded as an important means to reduce the environmental stress induced by transport, and as beneficial for the economy and public health. Scientific evidence for the positive effects of bicycling is growing, but these facts by themselves of course do not effectuate the societal change desired by advocacy organizations and policymakers. Their efforts to propagate and stimulate bicycling are an important driver for bicycle research, and is reflected in one of the main questions the field is currently focusing on: to understand why people bicycle or take up bicycling.

To help answer this question, many studies in the relatively young yet exponentially growing field of bicycle research (Pucher \& Buehler, 2017) seek to model the relation between potential explanatory factors, like social background, attitude towards cycling, travel distance, infrastructure quality, and bicycle practice (e.g., Aldred \& Jungnickel, 2014; Gatersleben \& Appleton, 2007; Heinen, Maat, \& van Wee, 2011; Moudon et al, 2005; Buehler \& Dill, 2016).

For practitioners, these studies provide a type of results that is easily implemented in policymaking, giving clear information on which factors encourage bicycle uptake. But the question is what applied value these empirics entail in terms of generalizability, and perhaps more importantly, transferability, since the research field is only at the beginning of a scientific understanding of bicycle practice.

\footnotetext{
${ }^{1}$ This article was written as a reflection of the Scientists for Cycling colloquium of Velo-city, 12 June, 2017, at Nijmegen https://www.velo-city2017.com/extracurricular/scientific-colloquium. The author holds a $\mathrm{PhD}$ in traffic and transport and currently works as an independent science writer at TerraCognita in the Netherlands (www.leoniewalta.nl).
}

Copyright 2018 Leonie Walta http://dx.doi.org/10.5198/jtlu.2018.1458

ISSN: 1938-7849 | Licensed under the Creative Commons Attribution - Noncommercial License 4.0

The Journal of Transport and Land Use is the official journal of the World Society for Transport and Land Use (WSTLUR) and is published and sponsored by the University of Minnesota Center for Transportation Studies. 


\section{Transferability}

In their quest to increase this understanding, bicycle researchers find that factors that are influential in a certain location or country may not be influential elsewhere. Popular press often depicts the "Dutch model" as a blueprint for bicycle planning, yet separate bike lanes with red tarmac have not automatically drawn people into bicycling in the rest of the world. Researchers are quick to point out that high quality bicycle lanes are indeed not the only factor that explains the high levels of bicycling. Universal, context-independent laws regarding bicycle uptake are not expected to exist, cultural differences play a large role. In countries like Brazil and Bangladesh bicycling at a young age is considered a "boys thing," while in the Netherlands women make more, but shorter, bicycle trips than men (Hoque, Mahmud, \& Qazi, 2008; Harms, Bertolini, \& te Brömmelstroet, 2014). Furthermore, bicycling is often associated with social stigma and disparaged social identities (Aldred, 2013; Jones \& de Azevedo, 2013). In developing nations, people link bicycling to low income and affordability — if you have any money you will drive a car - while in developed nations cycling may be labelled as part of negative moral discourse, for example as a form of "irresponsible parenting."

Furthermore, the success of policies is bigger in locations where bicycling is already an established mode of transport, including a network of bicycle infrastructure, than in locations where there merely is a desire for better bicycling facilities by policymakers and bicycling advocates. For example, a new stretch of infrastructure is most likely to be used in a place where bicycle uptake is already high.

\section{$3 \quad$ Methodology}

Next to their inability to retrieve generalized results, researchers struggle in establishing causal relations between determinant factors and bicycle practice. Methodological requirements, the nature of the subject and research funds are at odds with each other.

From cross-sectional data, that are often used in this field, only correlations can be inferred. For instance, it can be established from data that people who like bicycling are found more often to be bicycling than people who don't like bicycling. But this does not prove that changing someone's attitude will encourage bicycling. Research using longitudinal data shows that causality can also work the other way around people who bicycle more develop a more positive attitude towards bicycling. And, while people with a healthy lifestyle are found to be more likely to cycle, they are not necessarily healthy because they are cycling, which is an example of self-selection (Cao, 2015; Kroesen, Handy, \& Chorus, 2017). Similarly, a stretch of new infrastructure may have attracted bicyclists, because it was them who asked for it to be built. Because of this reversed causality, correlations often overestimate the actual effect of factors, summing up the effects in both directions.

One possible designated methodology to determine causality are randomized controlled experiments, such as used in trials for new drugs. The strict rules for research design make experiments virtually impossible in bicycle research or at least astronomically expensive. This can be partially overcome if a natural experiment occurs. For example, if one city implements a cycling policy, while another city, with a comparable population, keeps things as it is. Such cases are rare, but present a valuable opportunity for systematic research.

Observational studies, before and after studies around the introduction of bicycle policy measures that are sometimes wrongly referred to as natural experiments offer an opportunity for longitudinal studies. Often funding issues get in the way of good and timely before studies. And causal relations are hard to infer, because of the many circumstantial factors. 
This can be overcome to some extent by a case-crossover design, as applied by Teschke et al. (2012) to study the safety of fourteen different infrastructure types. This design compared the location on the road where people were injured with another, randomly chosen, location during the same trip. In this way, the researchers did not have to control for factors as age, gender, risk-taking behavior, traffic conditions, and weather; the subjects were their own control.

\section{$4 \quad$ Causality}

While some researchers are seeking sophisticated methodological ways to get closer to causal relations between determinant factors and bicycle practice, others comment that this approach is founded on a wrong conceptualization of causality. These latter researchers argue that the behavior of people cannot be reduced to a cause and effect chain, with certain causes having a high probability to lead to a certain effect. Instead they assume that actions taken in the real world, can trigger multiple responses, with unknown probabilities (see for instance Kroesen et al., 2017). This conceptualization of human behavior and the methodology to investigate it are rooted in social sciences. An example of such a methodology is to provide infrastructure and rules, designed to provide the right conditions for desirable cycling practices, and study the emerging behavior.

Furthermore, some researchers argue that bicycling is a cultural phenomenon, and that changes in culture are much more influential than changes in individual determinant factors.

\section{$5 \quad$ Moving forward}

A way of moving forward in bicycle research may be to reconsider the application of methods rooted in the natural science approach, while it is more likely that a scientifically sound understanding of bicycle practice can be derived based on social science methodology. At the same time, deriving a more scientific answer to the question of why people bicycle may compromise the usability of the outcomes for policymaking practice, and contributing to societal change remains an important driving force behind bicycle research.

Another way of moving forward is to acknowledge local differences and aim for a scope of research that is as universal as possible, while being as local as necessary. While the interest to extrapolate successful practices is understandable, the importance to realize local potential and precincts should not be undermined.

\section{Acknowledgements}

The author would like to thank Fariya Sharmeen, Kevin Krizek and Karel Martens for their suggestions and proposed references. 


\section{References}

Aldred, R. (2013). Incompetent or too competent? Negotiating everyday cycling identities in a motor dominated society. Mobilities, 8(2), 252-271.

Aldred, R., \& Jungnickel, K. (2014). Why culture matters for transport policy: The case of cycling in the UK. Journal of Transport Geography, 34, 78-87.

Buehler, R., \& Dill, J. (2016). Bikeway networks: A review of effects on cycling. Transport Reviews, $36(1), 9-27$.

Cao, X. (2015). Examining the impacts of neighborhood design and residential self-selection on active travel: A methodological assessment. Urban Geography, 36(2), 236-255.

Kroesen, M., Handy, S., \& Chorus, C. (2017). Do attitudes cause behavior or vice versa? An alternative conceptualization of the attitude-behavior relationship in travel behavior modeling. Transportation Research Part A: Policy and Practice, 101, 190-202.

Gatersleben, B., \& Appleton, K. M. (2007). Contemplating cycling to work: Attitudes and perceptions in different stages of change. Transportation Research Part A: Policy and Practice, 41(4), 302-312.

Harms, L., Bertolini, L., \& te Brömmelstroet, M. (2014). Spatial and social variations in cycling patterns in a mature cycling country: Exploring differences and trends. Journal of Transport and Health, 1(4), 232-242.

Heinen, E., Maat, K., \& Van Wee, B. (2011). The role of attitudes toward characteristics of bicycle commuting on the choice to cycle to work over various distances. Transportation Research Part D: Transport and Environment, 16(2), 102-109.

Hoque, M. M., Mahmud, S. S., \& Qazi, A. S. (2008) Cycling in Bangladesh. Bicycling in Asia, 81-89. Jones, T., \& de Azevedo, L. N. (2013). Economic, social and cultural transformation and the role of the bicycle in Brazil. Journal of Transport Geography, 30, 208-219.

Moudon, A. V., Lee, C., Cheadle, A. D., Collier, C. W., Johnson, D., Schmid, T. L., \& Weather, R. D. (2005). Cycling and the built environment, a US perspective. Transportation Research Part D: Transport and Environment, 10(3), 245-261.

Pucher, J., \& Buehler, R. (2017). Cycling towards a more sustainable transport future. Transport Reviews, 37(6), 689-694.

Teschke, K., Harris, M. A., Reynolds, C. C. O., Winters, M., Babul, S., Chipman, M., ... Cripton, P. A. (2012). Route Infrastructure and the risk of injuries to bicyclists: A case-crossover study. American Journal of Public Health, 102(12), 2336-2343. http://doi.org/10.2105/AJPH.2012.300762 